\title{
EFFECTS OF STENOSIS AND POST STENOTIC DILATATION ON JEFFREY FLUID FLOW IN ARTERIES
}

\author{
K.Maruthi Prasad ${ }^{1}$, R.Bhuvana Vijaya ${ }^{2}$, C.Umadevi ${ }^{3}$ \\ ${ }^{\text {I}}$ Department of Mathematics, GITAM University, Hyderabad Campus, Telangana, India \\ ${ }^{2}$ Department of Mathematics, JNTU College of Engineering, Anantapur, A.P, India \\ ${ }^{3}$ Department of Mathematics, TKR College of Engineering, Hyderabad, Telangana, India
}

\begin{abstract}
The steady flow of Jeffrey fluid through a tube with both constrictions and dilatations have been studied. The expressions for velocity, pressure drop, volumetric flow rate, resistance to the flow, wall shear stress have been derived by assuming that the stenosis is mild. It is found that the resistance to the flow increases with the height of the stenosis and decreases with post stenotic dilatation. The pressure drop, velocity and shear stress at the walls decrease with the height of stenosis and Jeffrey fluid parameter, but the same parameters increase with the post stenotic dilatation.
\end{abstract}

Keywords: Jeffrey fluid, stenosis, dilatation, Jeffrey fluid parameter, wall shear stress.

\section{INTRODUCTION}

In the present world, diseases related to the heart are the main causes of death. In arteries, the flow characteristics of blood can be altered by arterial diseases such as stenosis and aneurysm. Stenosis is caused due to intravascular atherosclerotic plaque which causes the narrowing of an artery. The intravascular atherosclerotic develops, at the arterial wall and protrudes into the lumen of the vessel. There occurs a significant change in blood flow, if an obstruction develops in the arteries. The predominant changes that occur are, change in pressure, shear stress at the walls and the impedance. A proper knowledge of the flow in the stenosed artery is required to understand the flow characteristics in a better way.

In view of this, several researchers have studied the flow of blood through arteries. Shukla et al., (1980) studied the behavior of non-Newtonian fluid flow of the blood in a stenosed artery. Biswas and Chakraborty (2010) investigated the influence of body acceleration and slip velocity at the wall by considering the blood as a two-fluid model. Singh et al. (2010) have developed a model through radially nonsymmetric artery.

Mishra and Verma (2010) investigated the flow of blood through a stenosed artery with uniform cross section. Gupta et al. (2012) studied the multiple stenoses effects with viscosity variation for power law fluid model. Sreenadh et al. (2011) developed a model and studied the multiple stenoses influence through an inclined tube of non-uniform cross section.

Hayat et al.,(2006) studied the peristaltic flow through a tube with an endoscope. Abd-Alla et al., (2014) observed the peristaltic flow in an asymmetric channel by considering the magnetic field and gravity field. Sudhakar reddy et al.,
(2012) studied viscosity effect on peristaltic flow of Jeffrey fluid in a uniform tube.

The above literature is concerned with different shapes stenosis with uniform or non-uniform cross sectional tubes by considering a non-Newtonian fluid.

A study conducted on post-stenotic dilatation by considering blood as Casson fluid (Tandon,et al.,1993). Pincombe et al.(1997), A.K. Singh and D.P.Singh (2012) studied the effects of post-stenotic dilatation by treating the fluid as Bingham fluid.

With this motivation, an effort is made in the present paper, to study the influence stenosis and post- stenotic dilatation on Jeffrey fluid. 


\section{MATHEMATICAL FORMULATION:}

Consider the flow of Jeffrey fluid through an axisymmetric artery containing multiple abnormal segments as shown in figure 1 .

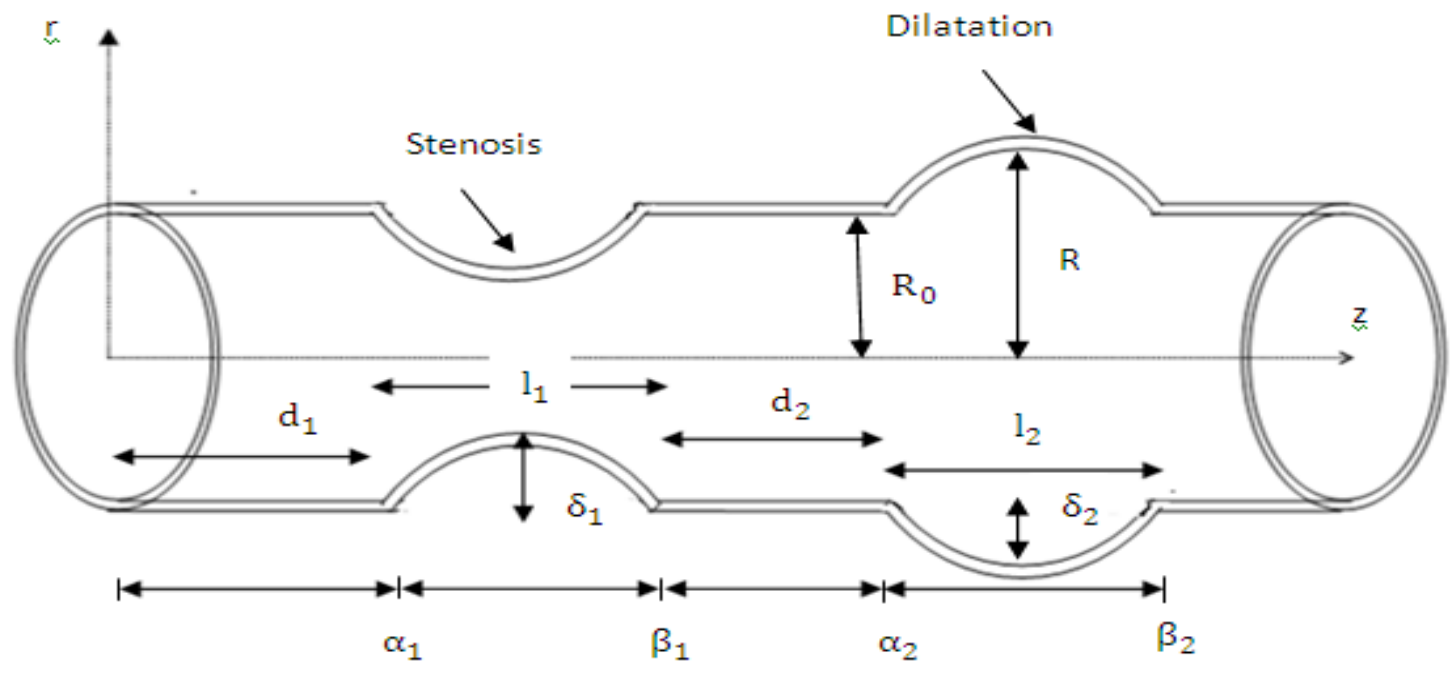

Fig.1. Geometry of arterial segment under consideration.

The equations describing the geometry of the wall, as shown in Fig. 1, are

$$
\left\{\begin{array}{l}
1-\frac{\delta_{i}}{2 R_{0}}\left[1+\operatorname{Cos} \frac{2 \pi}{l_{i}}\left(z-\alpha_{i}-\frac{l_{i}}{2}\right), \text { for } \alpha_{i} \leq z \leq \beta_{i}\right. \\
h=\frac{R(z)}{R_{0}}=1 \quad, \text { Otherwise }
\end{array}\right.
$$

Where $\delta_{i}$ represents the maximum distance of the $i^{\text {th }}$ abnormal segment, $R$ represents the radius of the artery, $R_{0}$ represents the radius of the normal artery, $l_{i}$ represents the length of the $i$ th abnormal segment, $\alpha_{i}$ represents the distance from the origin to the start of the $i$ th abnormal segment and is given by

$$
\alpha_{i}=\left(\sum_{j=1}^{i}\left(d_{j}+l_{j}\right)\right)-l_{i}
$$

And $\beta_{i}$ represents the distance from the origin to the end of the $i^{\text {th }}$ abnormal segment

$$
\beta_{i}=\left(\sum_{j=1}^{i}\left(d_{j}+l_{j}\right)\right)
$$

Where $d_{i}$ represents the distance separating the start of the $i^{\text {th }}$ abnormal segment from the end of the $i-1$ th, or from the start of the segment if $i=1$.

The constitutive equations for the fluid are (M.Sudhakar Reddy et al. (2012))

$$
T=-p I+S
$$

$$
S=\frac{\mu}{1+\lambda_{1}}\left(\frac{\partial \gamma}{\partial t}+\lambda_{2} \frac{\partial^{2} \gamma}{\partial t^{2}}\right)
$$

Where $T$ represents the Caushy stress tensor, $S$ represents the extra stress tensor, $p$ represents the pressure, $I$ represents the identity tensor, $\lambda_{1}$ represents the ratio of the relaxation to retardation times, $\lambda_{2}$ represents the retardation time, $\mu$ represents the dynamic viscosity and $\bar{\gamma}$ represents the shear rate.

The equations governing to the steady incompressible Jeffrey fluid are

$\frac{\partial u}{\partial r}+\frac{u}{r}+\frac{\partial w}{\partial z}=0$

$\rho\left(u \frac{\partial}{\partial r}+w \frac{\partial}{\partial z}\right) u=-\frac{\partial p}{\partial r}+\frac{1}{r} \frac{\partial}{\partial r}\left(r S_{r r}\right)+\frac{\partial}{\partial z}\left(S_{r z}\right)-\frac{S_{\theta \theta}}{r}$

$\rho\left(u \frac{\partial}{\partial r}+w \frac{\partial}{\partial z}\right) w=-\frac{\partial p}{\partial z}+\frac{1}{r} \frac{\partial}{\partial r}\left(r S_{r z}\right)+\frac{\partial}{\partial z}\left(S_{z z}\right)$

Where

$$
\begin{aligned}
& S_{r r}=\frac{2 \mu}{1+\lambda_{1}}\left(1+\lambda_{2}\left(u \frac{\partial}{\partial r}+w \frac{\partial}{\partial z}\right) \frac{\partial u}{\partial r}\right. \\
& S_{r z}=\frac{\mu}{1+\lambda_{1}}\left(1+\lambda_{2}\left(u \frac{\partial}{\partial r}+w \frac{\partial}{\partial z}\right)\right)\left(\frac{\partial w}{\partial r}+\frac{\partial u}{\partial z}\right) \\
& S_{z z}=\frac{2 \mu}{1+\lambda_{1}}\left(1+\lambda_{2}\left(u \frac{\partial}{\partial r}+w \frac{\partial}{\partial z}\right) \frac{\partial w}{\partial z}\right. \\
& S_{\theta \theta}=\frac{2 \mu}{1+\lambda_{1}}\left(1+\lambda_{2}\left(u \frac{\partial}{\partial r}+w \frac{\partial}{\partial z}\right) \frac{u}{r}\right.
\end{aligned}
$$


Introducing the following non dimensional variables

$\bar{z}=\frac{z}{L}, \bar{\delta}=\frac{\delta}{R_{0}}, \bar{R}=\frac{R}{R_{0}}, \bar{P}=\frac{P}{\frac{\mu U L}{R_{0}^{2}}}, \bar{u}=\frac{u}{U}, \bar{w}=\frac{L}{U \delta} w, R e=$

$\frac{\rho R_{0} U}{\mu}, \bar{\mu}=\frac{\mu}{\mu_{0}}$

Where $U$ represents the average velocity.

Eqs. (6)- (9), under the assumptions of low Reynolds number and mild stenosis $\frac{\delta}{R_{0}} \ll 1$ takes the form (after dropping the bars)

$\frac{\partial u}{\partial r}+\frac{u}{r}+\frac{\partial w}{\partial z}=0$

$\frac{\partial p}{\partial r}=0$

$-\frac{\partial p}{\partial z}=\frac{1}{1+\lambda_{1}} \frac{1}{r} \frac{\partial}{\partial r}\left(r \frac{\partial u}{\partial r}\right)$

The corresponding non dimensional boundary conditions are

$$
\begin{gathered}
\frac{\partial u}{\partial r}=0 \text { at } r=0 \\
u=0 \text { at } r=h
\end{gathered}
$$

\section{SOLUTION OF THE PROBLEM:}

Solving the equation (12) for , using the eqs. (13) and (14), yields

$u=\left(\frac{h^{2}-r^{2}}{4}\right)\left(1+\lambda_{1}\right) \frac{d p}{d z}$

The volumetric flow rate is defined by

$Q=2 \pi \int_{0}^{h} u r d r$

On integrating,

$Q=\frac{\pi h^{4}}{8}\left(1+\lambda_{1}\right) \frac{d p}{d z}$

$\frac{d p}{d z}=\frac{8 Q}{\pi h^{4}\left(1+\lambda_{1}\right)}$

When $\lambda_{1}=0$, the equations indicates the Newtonian fluid The pressure drop $\Delta p$ across the stenosis between $z=0$ to $z=1$ is obtained by integrating Eq. (18), as

$\Delta p=\int_{0}^{1} \frac{d p}{d z} d z$

$\Delta p=\int_{0}^{1} \frac{8 Q}{\pi h^{4}\left(1+\lambda_{1}\right)} d z$

the resistance to the flow, $\lambda$, is defined by

$\lambda=\frac{\Delta p}{Q}=\frac{1}{Q} \int_{0}^{1}\left(\frac{8 Q}{\pi h^{4}\left(1+\lambda_{1}\right)}\right) d z$
The pressure drop in the absence of stenosis $(h=1)$ is denoted by $\Delta p_{\mathrm{N}}$, is obtained from Eq. (18).

$\Delta P_{N}=\int_{0}^{1} \frac{8 Q}{\pi\left(1+\lambda_{1}\right)} d z$

The resistance to the flow in the normal artery is denoted by $\lambda_{\mathrm{N}}$ is obtained from Eq. (22),

as $\quad \lambda_{\mathrm{N}}=\frac{\Delta P_{N}}{Q}=\frac{1}{Q} \int_{0}^{1} \frac{8 Q}{\pi\left(1+\lambda_{1}\right)} d z$

The normalized resistance to the flow denoted by

$\bar{\lambda}=\frac{\lambda}{\lambda_{N}}$

The wall shear stress is of the form

$S_{r z}=\left.\left\{\frac{1}{1+\lambda_{1}}\left[\frac{\partial u}{\partial r}+\lambda_{2} u\left(\frac{\partial^{2} u}{\partial r \partial z}\right)\right]\right\}\right|_{r=h}$

\section{RESULTS AND ANALYSIS}

The velocity $(u)$, volumetric flow $\operatorname{rate}(Q)$, impedance $\bar{\lambda}$ and shear stress at the wall $\left(\tau_{\mathrm{h}}\right)$ are defined in the equations 15 , 17,24 and 25 respectively. By considering $d_{1}=0.2, d_{2}=$ $0.2, L_{1}=L_{2}=0.2, L=1, Q=0.1$, the effects of various parameters on the resistance to the flow $(\bar{\lambda})$, pressure drop $(\Delta p)$, wall shear stress $\left(\tau_{\mathrm{h}}\right)$ are calculated and shown in the Figs. 2-17.

It is observed that with the height and length of the stenosis, the resistance to the flow increases, but it decreases with the height and length of the post stenotic dilatation (Figs. 2-6).

It is noticed that pressure drop increases with the height of the stenosis and volumetric flow rate, but decreases with Jeffrey fluid parameter. Figs.7\&8, 10\&11.Similarly, in post stenotic dilatation it decreases with the volumetric flow rate and increases with Jeffrey fluid parameter (Figs.9\&12).

It is observed the velocity decreases with stenosis height and Jeffrey fluid parameter. Fig.13 but it increases with post stenotic dilitation and Jeffrey fluid parameter Fig. 14.

It is observed that the shear stress at walls decreases with the post stenotic dilatation and increases with Jeffrey fluid parameter $\lambda_{1}$ (Figs.15-16), but it increases with the height of stenosis, and decreases with the Jeffrey fluid parameter $\lambda_{1}$ (Fig.17). 


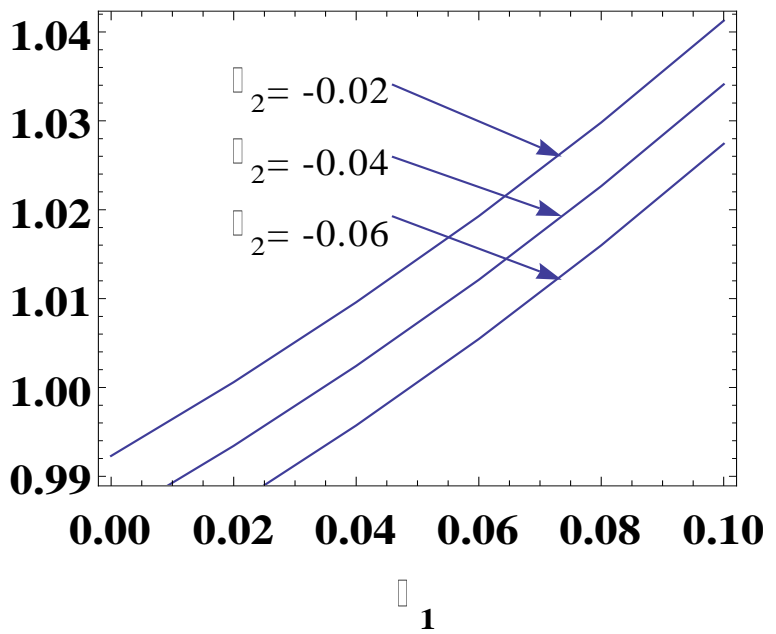

Fig2: Variation of flow resistance $\bar{\lambda}$ with $\delta_{1}$ for different $\delta_{2}\left(d_{1}=0.2, d_{2}=0.2, L_{1}=L_{2}=0.2, L=1, Q=0.1, \lambda_{1}=0.2\right)$

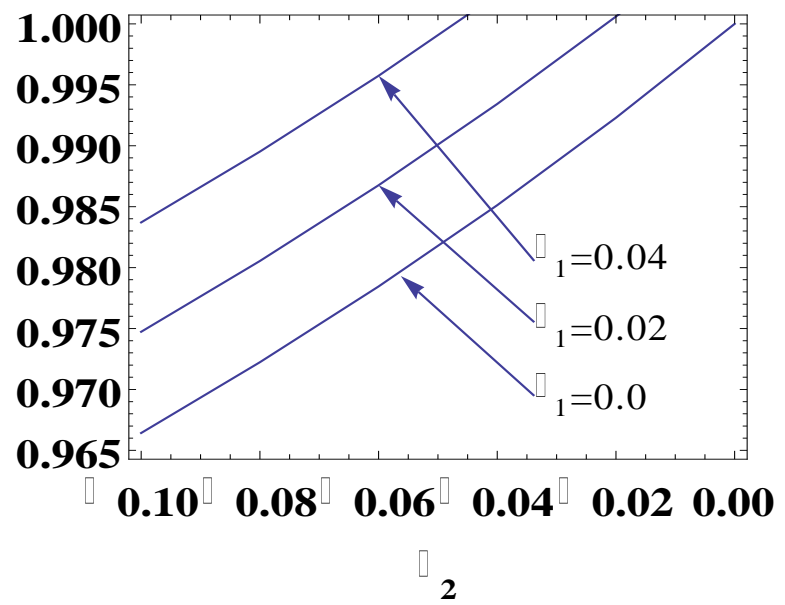

Fig3: Variation of impedance $\bar{\lambda}$ with $\delta_{2}$ for different $\delta_{1}\left(d_{1}=0.2, d_{2}=0.2, L_{1}=L_{2}=0.2, L=1, Q=0.1, \lambda_{1}=0.2\right)$

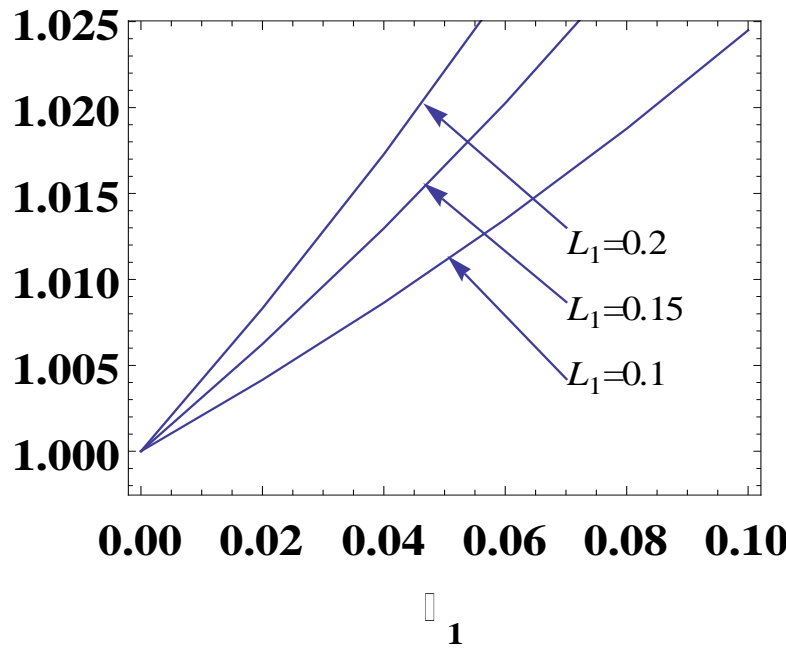

Fig4: Variation of impedance $\bar{\lambda}$ with $\delta_{1}$ for different $L_{1}$ $\left(d_{1}=0.2, d_{2}=0.2, L_{2}=0.2, L=1, Q=0.1, \delta_{2}=0.0, \lambda_{1}=0.2\right)$

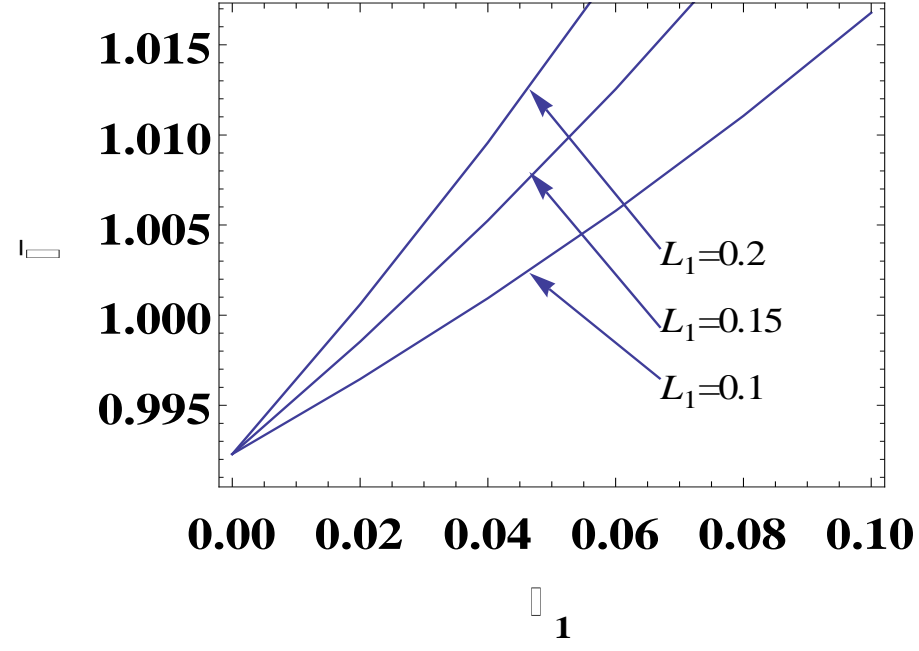

Fig5: Variation of impedance $\bar{\lambda}$ with $\delta_{1}$ for different $\mathrm{L}_{1}$ $\left(d_{1}=0.2, d_{2}=0.2, L_{2}=0.2, L=1, Q=0.1, \delta_{2}=-0.02, \lambda_{1}=0.2\right)$

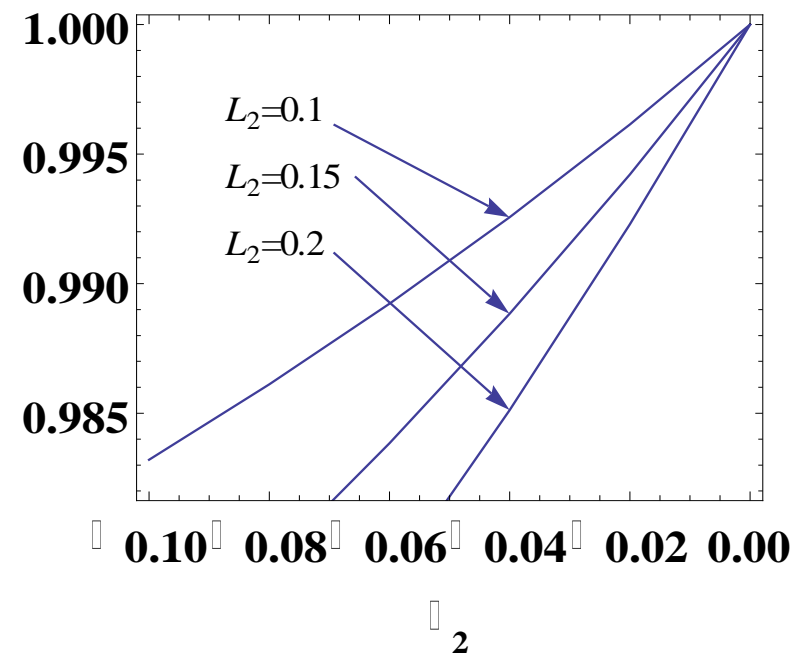

Fig6: Variation of impedance $\bar{\lambda}$ with $\delta_{2}$ for different $\mathrm{L}_{2}$ $\left(d_{1}=0.2, d_{2}=0.2, L_{1}=0.2, L=1, Q=0.1, \delta_{1}=0, \lambda_{1}=0.2\right)$

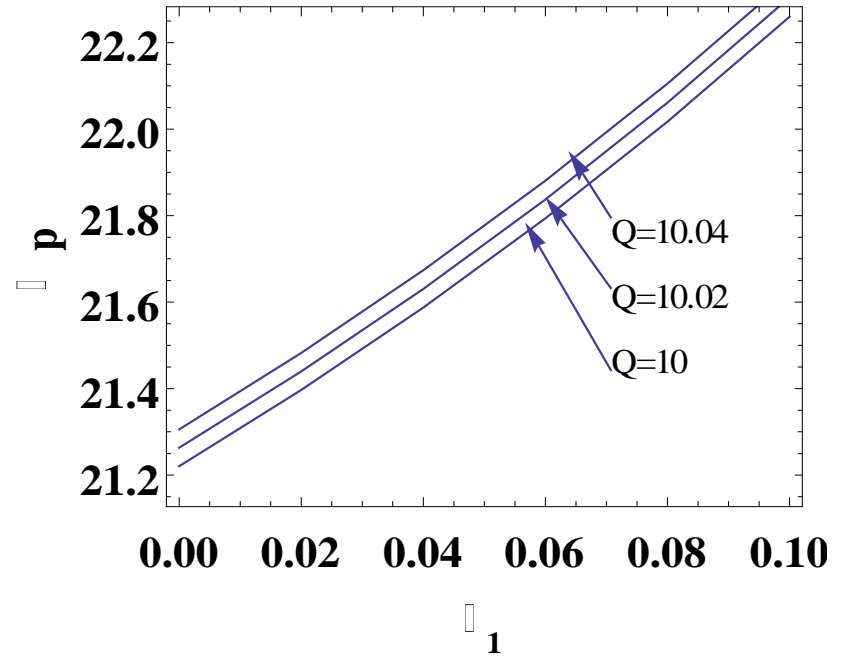

Fig7: Variation of pressure drop $\Delta p$ with $\delta_{1}$ for different Q $\left(d_{1}=0.2, d_{2}=0.2, L_{1}=L_{2}=0.2, L=1, \lambda_{1}=0.2, \delta_{2}=0\right)$ 


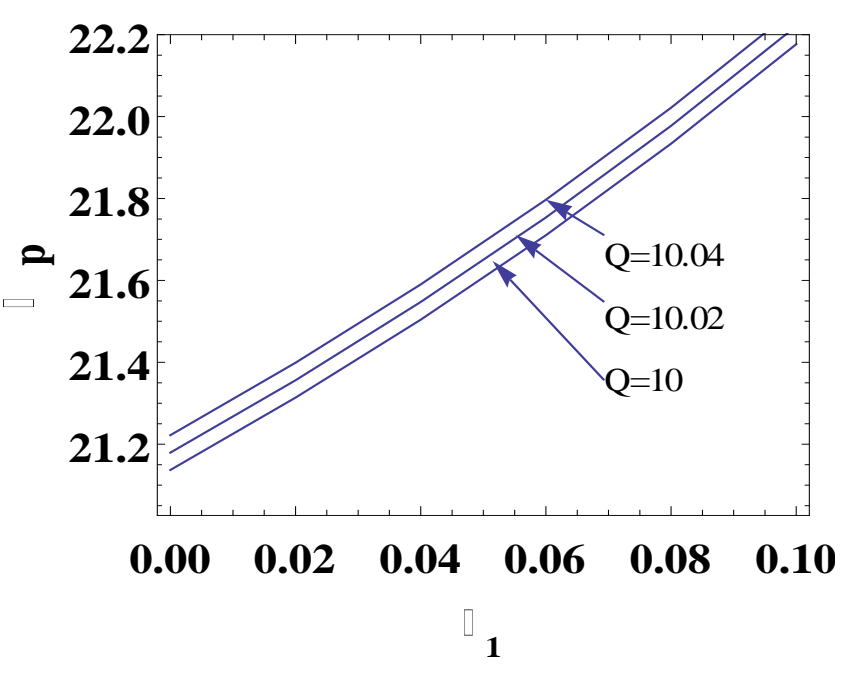

Fig8: Variation of pressure drop $\Delta p$ with $\delta_{1}$ for different $\mathrm{Q}$ $\left(d_{1}=0.2, d_{2}=0.2, L_{1}=L_{2}=0.2, L=1, \lambda_{1}=0.2, \delta_{2}=-0.01\right)$

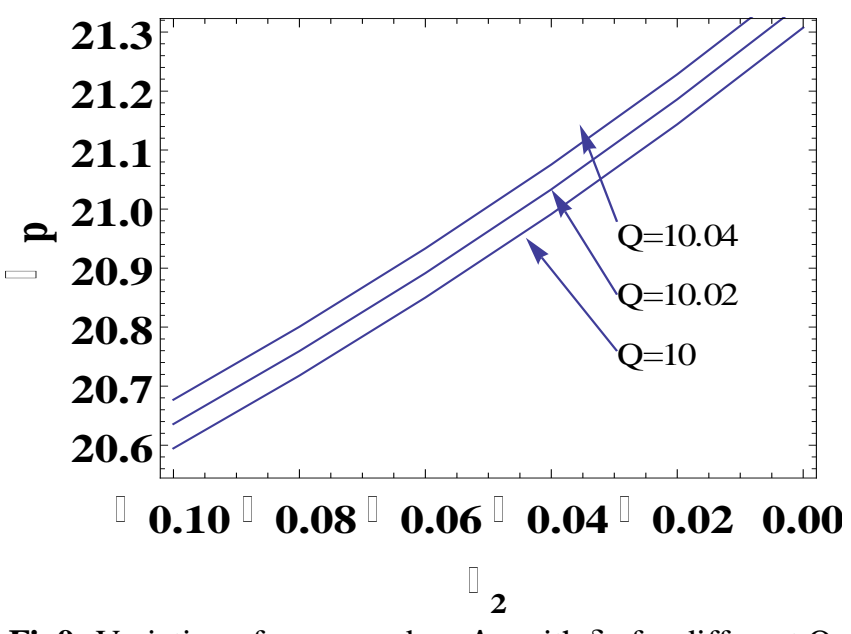

Fig9: Variation of pressure drop $\Delta p$ with $\delta_{2}$ for different Q $\left(d_{1}=0.2, d_{2}=0.2, L_{1}=L_{2}=0.2, L=1, \lambda_{1}=0.2, \delta_{1}=0.0\right)$

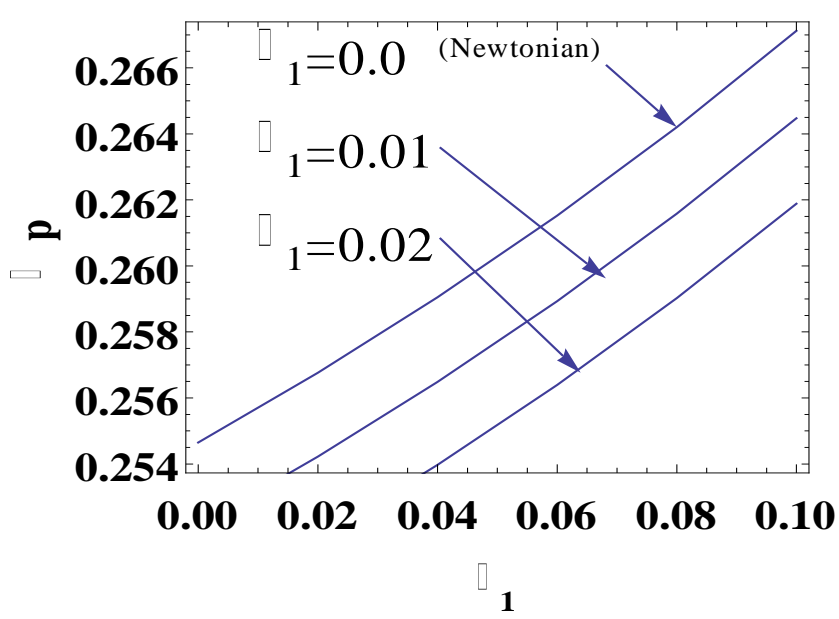

Fig10: Variation of pressure drop $\Delta p$ with $\delta_{1}$ for different $\lambda_{1}$ $\left(d_{1}=0.2, d_{2}=0.2, L_{1}=L_{2}=0.2, L=1, \mathrm{Q}=0.1, \delta_{2}=0.0\right)$

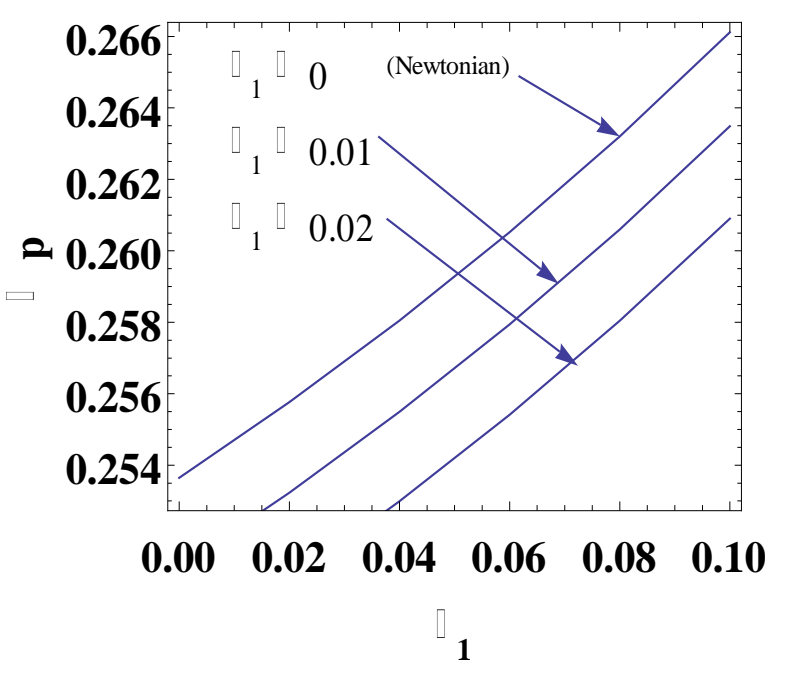

Fig11: Variation of pressure drop $\Delta p$ with $\delta_{1}$ for different $\lambda_{1}$ $\left(d_{1}=0.2, d_{2}=0.2, L_{1}=L_{2}=0.2, L=1, \mathrm{Q}=0.1, \delta_{2}=-0.01\right)$

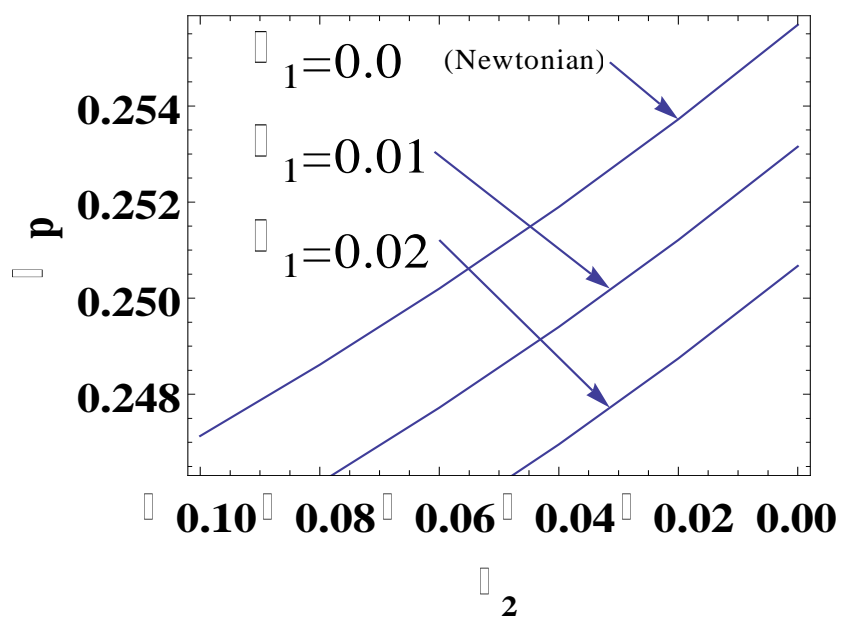

Fig12: Variation of pressure drop $\Delta p$ with $\delta_{2}$ for different $\lambda_{1}\left(d_{1}=0.2, d_{2}=0.2, L_{1}=L_{2}=0.2, L=1, \mathrm{Q}=0.1, \delta_{1}=0.01\right)$

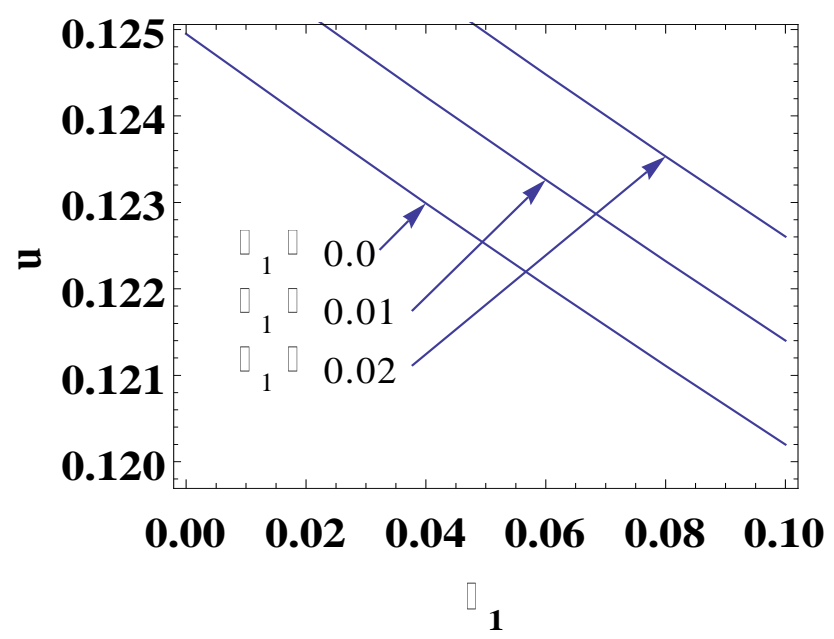

Fig13: Variation of velocity u with $\delta_{1}$ for different $\lambda_{1}$ $\left(\mathrm{Q}=0.1, \delta_{2}=0, r=0.02, P=0.1\right)$ 


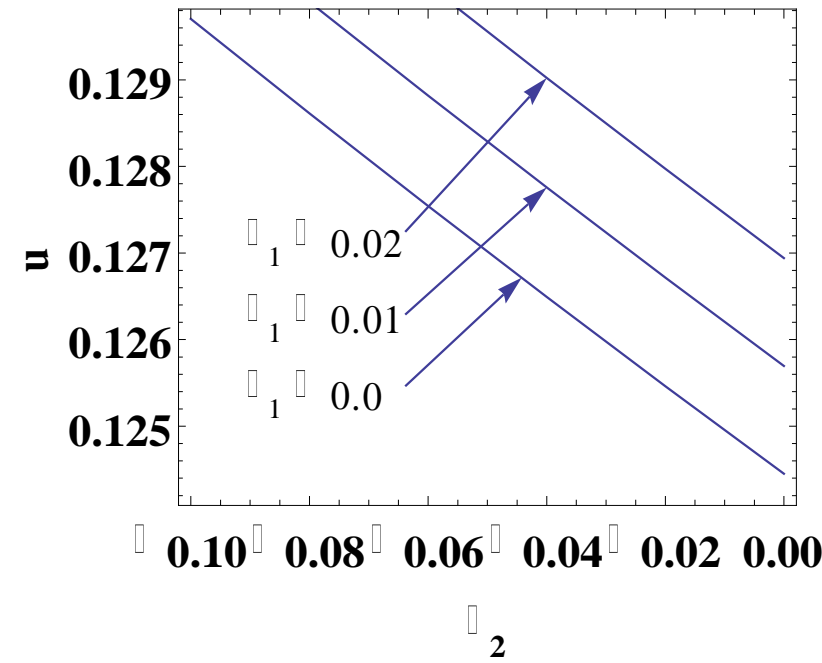

Fig14: Variation of velocity $\mathrm{u}$ with $\delta_{2}$ for different $\lambda_{1}$ $\left(\mathrm{Q}=0.1, \delta_{1}=0.01, r=0.02, P=0.1\right)$

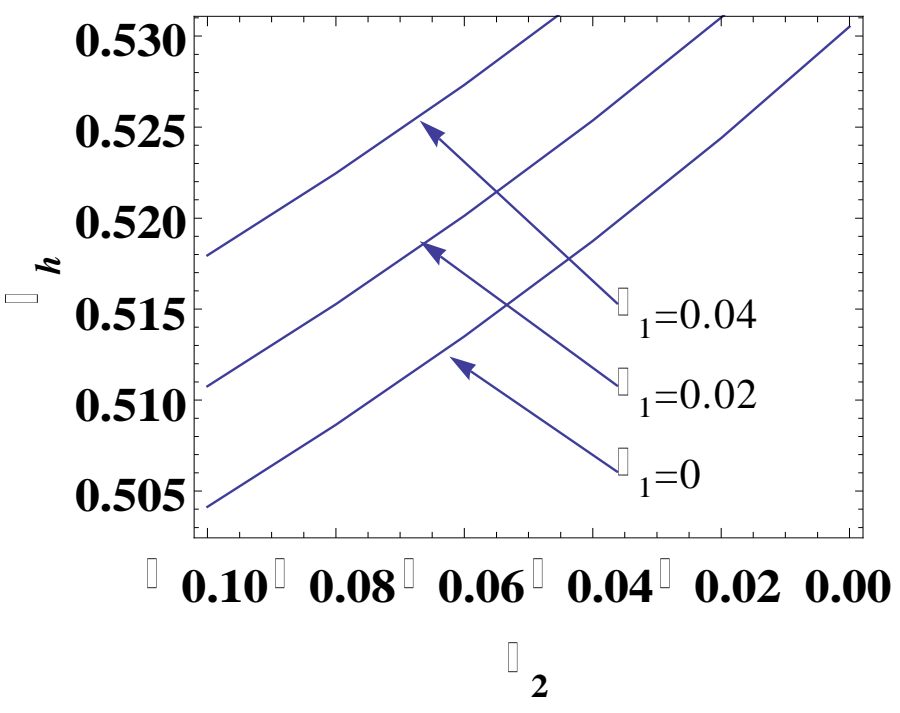

Fig15: Variation of wall shear stress $\tau_{\mathrm{h}}$ with $\delta_{2}$ for different $\delta_{1}\left(Q=0.1, \lambda_{1}=0.2\right)$

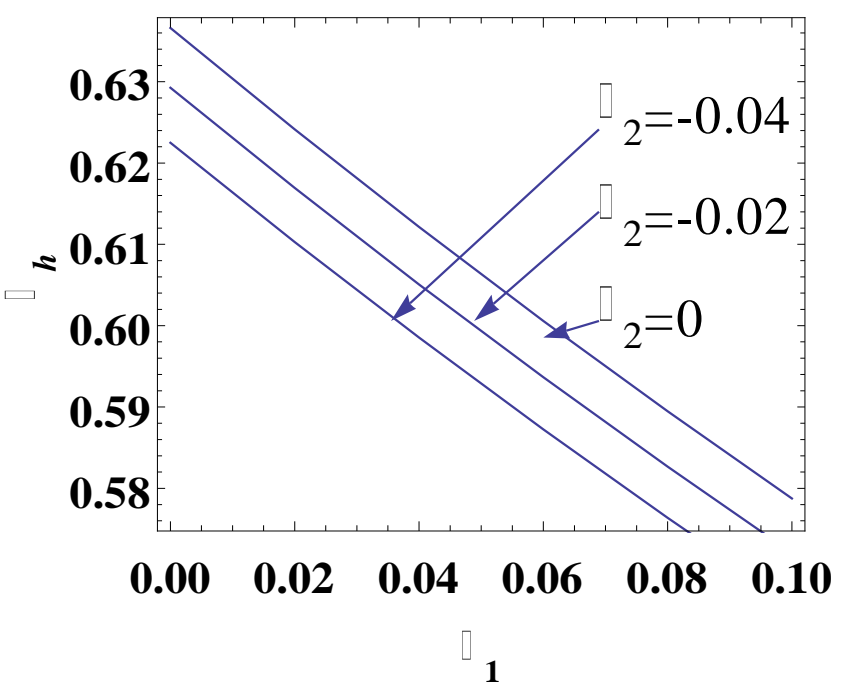

Fig16: Variation of shear stress at the walls $\tau_{\mathrm{h}}$ with $\lambda_{1}$ for different $\delta_{2}\left(\mathrm{Q}=0.1, \delta_{1}=0.0\right)$

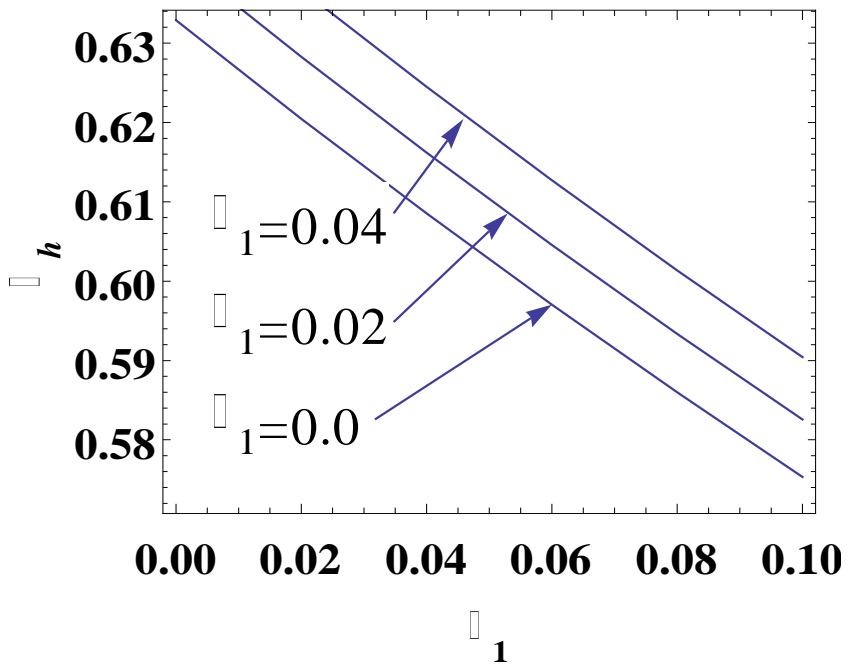

Fig17: Variation of shear stress at the walls $\tau_{\mathrm{h}}$ with $\lambda_{1}$ for different $\delta_{1}\left(\mathrm{Q}=0.1, \delta_{2}=0.01\right)$

\section{CONCLUSION}

A study on jeffrey fluid through stenosed arteries with post stenotic dilatation has been presented. Solutions have been obtained for mild stenosis and it has been shown that, the resistance to the flow increases as the height of stenosis increases but decreases with post stenotic dilitation. The pressure drop increases with the increase of volumetric flow rate with the height of stenosis but it decreases with post stenotic dilitation.The pressure drop and velocity decreases with the Jeffrey fluid parameter as the stenosis height increases, but they increase with post stenotic dilatation. The shear stress at the walls increase with the stenosis height and decreases with Jeffrey fluid parameter, but in post stenotic dilatation, it decreases with Jeffrey fluid parameter.

\section{REFERENCES}

[1] J.B. Shukla, R.S. Parihar andB.R.P. Rao , "Effects of stenosison non-Newtonain flow of the blood in an artery", Bull.Math. Biol.42, pp.283-294, 1980.

[2] A.M. Abd-Alla, S.M. Abo-Dahab and M.M. Albalawi, "Magnetic field and gravity effects on peristaltic transport of a Jeffrey fluid in an asymmetric channel", Abstract and Applied Analysis, Vol.2014,Article ID896121, 11 pages, 2014.

[3] T. Hayat, N.Ali, S.Asghar and A.M.Siddiqui, "Exact peristaltic flow in tubes with an endoscope", Applied Mathematics and Computation, Vol.182, no.1, pp.359-368, 2006.

[4] M. Sudhakar Reddy, M.V, Subba Reddy, B.Jayarami Reddy and S.Ramakrishna,"Effect of variable viscosity on the peristaltic flow of a Jeffrey fluid in a uniform tube", Advances in applied science research,Vol.3, no2, pp. 900-908, 2012.

[5] P.N. Tandon, U.V.Rana, M.Kawahara, V.K. Katiyar,"A model for blood flow through stenotic tube", Int. J.Biomed. Comput, 32,pp. 62-78, 1993.

[6] B. Pincombe and J.N. Mazumdar " The Effects of Post- Stenotic dilitations on the flow of a blood analogue through stenosed coronary arteries 
arteries”, Mathl.Comput.Modelling.Vol.25,no-6, pp.57-70, 1997.

[7] S. Gupta, M. Gupta and S.P. Singh "Effect of multiple stenosis with radial viscosity variation in an artery: Power law fluid model, International Transactions in Mathematical Sciences and Computer 5,1, pp. 75-93, 2012.

[8] B.K. Mishra and N. Verma "Effects of stenosis on non-Newtonian flow of blood in blood vessels", Australian Journal of Basic and Applied Sciences4, 4, pp. 588-601, 2010.

[9] B.Singh, P. Joshi and B.K. Joshi, "Blood flow through an artery having radially non-symmetric mild stenosis", Appl. Math. Sci., Vol. 4, pp. 10651072, 2010.

[10] D. Biswas and U.S. Chakraborty, "Two-Layered Pulsatile Blood Flow in a stenosed artery with body acceleration and slip at wall". Appl. Math., Vol 5, pp. 1401-1418, 2010.

[11] S. Sreenadh, A.R. Pallavi, B.H. Satyanarayana, "Flow of a Casson Fluid Through an Inclined Tube of Non-uniform Cross Section with Multiple Stenoses", Adv. Appl. Sci. Res. 2 (5), pp. 340-349, 2011.

[12] A.K. Singh and D.P. Singh, "A Computational study of Bingham plastic flow of Blood through an artery by multiple stenoses and post dilatation", Adv. Appl. Sci. Res. 3(5), pp.3285-3290, 2012. 